Marquette University

e-Publications@Marquette

Physics Faculty Research and Publications

Physics, Department of

$11-1-1998$

\title{
Inhibition of the Aminopeptidase from Aeromonas proteolytica by l-Leucinephosphonic Acid, a Transition State Analogue of Peptide Hydrolysis
}

Brian Bennett

Marquette University, brian.bennett@marquette.edu

Richard C. Holz

Marquette University, richard.holz@marquette.edu

Accepted version. Journal of the American Chemical Society, Vol. 120, No. 46 (November 1998):

12139-12140. DOI. (C) 1998 American Chemical Society. Used with permission.

Brian Bennett and Richard C. Holz were affiliated with Utah State University at the time of publication. 


\title{
Inhibition of the Aminopeptidase from Aeromonas Proteolytica by l- Leucinephosphonic Acid, a Transition State Analogue of Peptide Hydrolysis
}

\author{
Brian Bennett \\ Department of Chemistry and Biochemistry, \\ Utah State University, \\ Logan, UH \\ Richard C. Holz \\ Department of Chemistry and Biochemistry, \\ Utah State University, \\ Logan, UH
}

Dinuclear metallohydrolases catalyze the degradation of many of the ubiquitous biomolecules in living organisms such as nucleic acids, phospholipids, and polypeptides..$^{1-4}$ They are therefore key players in carcinogenesis, tissue repair, and protein degradation processes. Several of these enzymes have been crystallographically characterized, but despite their ubiquity and the wealth of structural information available little is known about how these structural motifs 
relate to function. The importance of understanding the mechanism of action of dinuclear hydrolases is underscored by the recent observation that a eukaryotic dinuclear metallo-aminopeptidase is the target for the antitumor drugs ovalicin and fumagillin. 5,6 In addition, the naturally occurring peptide analogue inhibitor, bestatin, was recently shown to significantly decrease HIV infection by inhibiting leucine aminopeptidase activity. ${ }^{7}$ In an effort to gain insight into the structure of the tetrahedral transition state of peptide hydrolysis, we have explored the nature of the interaction of I-leucinephosphonic acid (LPA) with the aminopeptidase from Aeromonas proteolytica (AAP).

AAP is a small, monomeric enzyme ( $32 \mathrm{kDa}$ ) that is thermostable for several hours at $70^{\circ} \mathrm{C} .{ }^{8}$ AAP has been crystallographically characterized and contains a ( $\mu$-aqua) $(\mu-$ carboxylato)dizinc(II) core with a terminal carboxylate and histidine residue coordinated to each metal ion. ${ }^{9}$ Bennett and Holz recently demonstrated that metal binding to AAP occurs in a sequential fashion, highlighting the potential for the formation of heterodimetallic sites. ${ }^{10,11}$ The addition of LPA to [CoZn(AAP)], monitored by electronic absorption spectroscopy, results in an increase in molar absorbtivity from 58 to $68 \mathrm{M}^{-1} \mathrm{~cm}^{-1}$ with no shift in the $\lambda_{\max }$ value of $525 \mathrm{~nm}$ (Figure $1 \mathrm{~A})$. On the other hand, the addition of LPA to [ZnCo(AAP)] (Figure 1B) results in a clear shift in $\lambda_{\max }$ from 525 to $500 \mathrm{~nm}$ with a concomitant increase in the molar absorptivity to $63 \mathrm{M}^{-1} \mathrm{~cm}^{-1}$. The visible absorption spectra of [CoZn(AAP)]-LPA and [ZnCo(AAP)]-LPA are comparable to the cobalt-substituted angiotensin converting enzyme ( $525 \mathrm{~nm}, \varepsilon \sim 75 \mathrm{M}^{-1} \mathrm{~cm}^{-1}$ ) and the noncatalytic site of native carboxypeptidase $A\left(525 \mathrm{~nm}, \varepsilon \sim 65 \mathrm{M}^{-1} \mathrm{~cm}^{-1}\right)$, both of which are proposed to be five coordinate. ${ }^{12,13}$ However, the absorption spectrum of [ $\mathrm{ZnCo}(\mathrm{AAP})]$-LPA exhibits fine structure comprised of five absorption bands at 488, 517, 549, 579, and $601 \mathrm{~nm}$. Electronic absorption fine structure similar to that observed for [ZnCo(AAP)]-LPA is sometimes observed in severely distorted octahedral $\mathrm{Co}$ (II) species. ${ }^{14}$ Therefore, extensive distortions away from octahedral symmetry are likely responsible for the effects of LPA binding on the electronic absorption spectrum of [ZnCo(AAP)]. Taken together, these data suggest an interaction between LPA and each metal ion in the dinuclear catalytic center of AAP. 

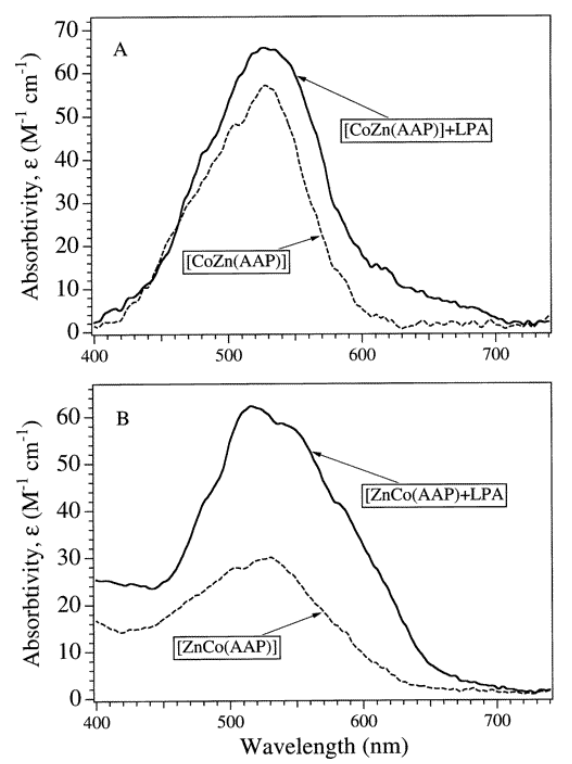

Figure 1 Electronic absorption spectrum of $(A)$ a $1 \mathrm{mM}$ buffered $(50 \mathrm{mM}$ Hepes; $\mathrm{pH}$ 7.5; 20\% 2-propanol) solution of [CoZn(AAP)] in the absence (dashed line) and presence (solid line) of 5 equiv of LPA, and (B) a $1 \mathrm{mM}$ buffered ( $50 \mathrm{mM}$ Hepes; $\mathrm{pH}$ 7.5; $20 \%$ 2-propanol) solution of [CoZn(AAP)] in the absence (dashed line) and presence (solid line) of 5 equiv of LPA. Electronic absorption spectra were recorded on a Shimadzu UV-3101PC spectrophotometer.

The EPR spectrum of resting [CoCo(AAP)] is shown in Figure $2 \mathrm{~A}$. The addition of LPA to [CoCo(AAP)] completely abolished the perpendicular mode EPR signal (Figure 2B). Examination of [CoCo(AAP)]-LPA in the parallel mode revealed an intense integer spin signal with a crossover point at $g \sim 10.3$ at $9 \mathrm{~K}$ suggesting that the two $S=3 / 2 \mathrm{Co}$ (II) ions are ferromagnetically coupled upon LPA binding. The signal exhibited maximum intensity at $4 \mathrm{~K}$ suggesting that it is a ground-state transition. Based on the reported magnetic properties of several $\mu$-aquo and $\mu$-hydroxo dicobalt(II) model complexes, weak to moderately strong antiferromagnetic coupling would be expected if LPA provided a single oxygen atom bridge. ${ }^{15,17}$ Moreover, bis( $\mu$-carboxylato)- and tetrakis( $\mu$-carboxylato)dicobalt(II) cores show weak antiferromagnetic spin coupling. ${ }^{18-20}$ These data suggest that a single oxygen atom bridge is not present in the [CoCo(AAP)]-LPA complex and that the ferromagnetic coupling is not likely mediated through the carboxylate bridge. Interestingly, when bestatin, which provides a single alkoxide bridging atom, is added to a sample of [CoCo(AAP)] no parallel or perpendicular mode EPR signals are observed suggesting that the two high-spin $\mathrm{Co}$ (II) ions are antiferromagnetically coupled. ${ }^{21}$ Bestatin likely binds to AAP in a 
fashion similar to d-iodophenylalanine hydroxamate which has been shown by X-ray crystallography to provide a single oxygen atom bridge between the two $\mathrm{Zn}$ (II) ions. ${ }^{22}$ Taken together, these data suggest that LPA and bestatin bind in different modes to the dinuclear Co(II) active site cluster of AAP.

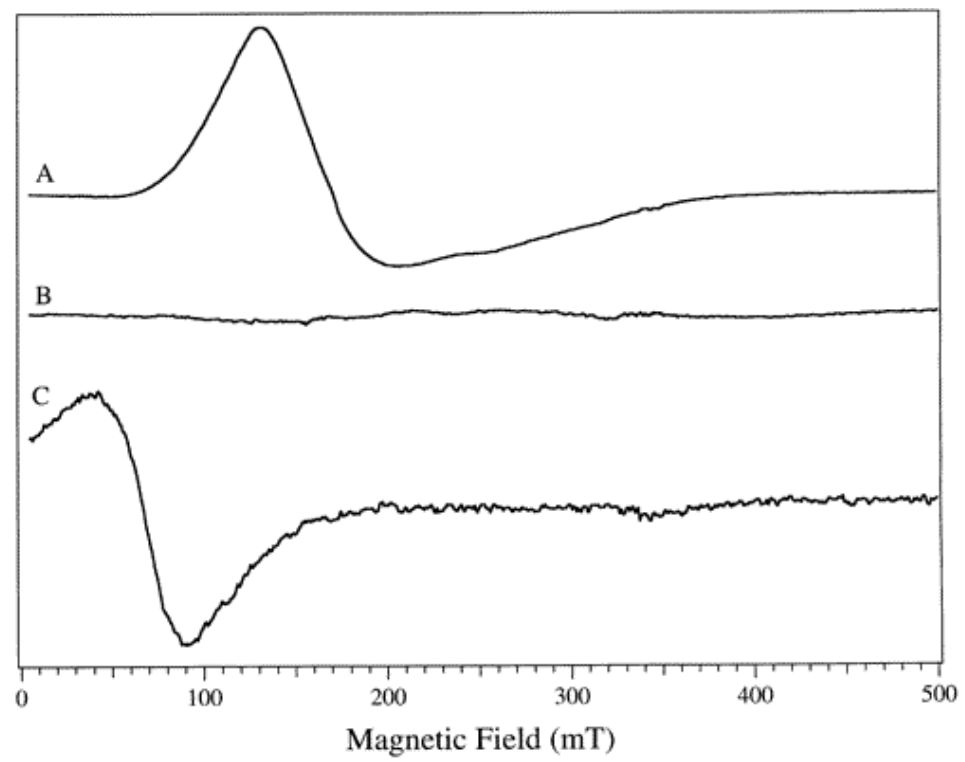

Figure 2 EPR spectra of a $1 \mathrm{mM}$ buffered solution of [CoCo(AAP)]: (A) perpendicular mode in the absence of LPA; (B) perpendicular mode in the presence of 5 equiv of LPA; and (C) parallel mode in the presence of 5 equiv of LPA. All samples were buffered with $50 \mathrm{mM}$ Hepes, pH 7.5, containing 20\% 2-propanol to prevent aggregation. All EPR spectra were recorded with a $1.26 \mathrm{mT}$ modulation amplitude and a sweep rate of $10 \mathrm{mT} \mathrm{s}^{-1}$. Perpendicular mode spectra were recorded at $10 \mathrm{~K}, 0.2 \mathrm{~mW}$ microwave power while parallel mode spectra were recorded at $3.9 \mathrm{~K}, 2 \mathrm{~mW}$ microwave power. All EPR spectra were recorded on a Bruker ESP-300E spectrometer equipped with an ER 4116 DM dual-mode cavity and an Oxford Instruments ESR-900 helium flow cryostat.

EPR studies of [CoZn(AAP)] and [ZnCo(AAP)] revealed that the two EPR signals observed in the resting enzymes, which arise from a $\mathrm{pH}$-dependent mixture of species, ${ }^{11}$ are replaced by signals due to a single species. For both [CoZn(AAP)]-LPA and [ZnCo(AAP)]-LPA a rhombic, ${ }^{59} \mathrm{Co}$ hyperfine split species is observed that could be simulated as a single species (Figure 3 ). ${ }^{15}$ These data indicate that the environments of the $\mathrm{Co}(\mathrm{II})$ ions in both [CoZn(AAP)] and [ZnCo(AAP)] become highly asymmetric and constrained upon the addition of LPA and clearly indicate interaction of LPA with both metal ions. Interestingly, the ${ }^{59} \mathrm{Co}$ hyperfine splitting for [ZnCo(AAP)]-LPA is significantly smaller than that observed for other Co(II)-substituted 
AAP species and suggests delocalization of some $30 \%$ of the paramagnetic electron density from the cobalt nucleus. This implies a direct interaction of the Co(II) ion with an electrophilic moiety such as the $\mathrm{N}$-terminal amine group of LPA which is also consistent with the anisotropy of $g_{\text {real }}$ for this species.

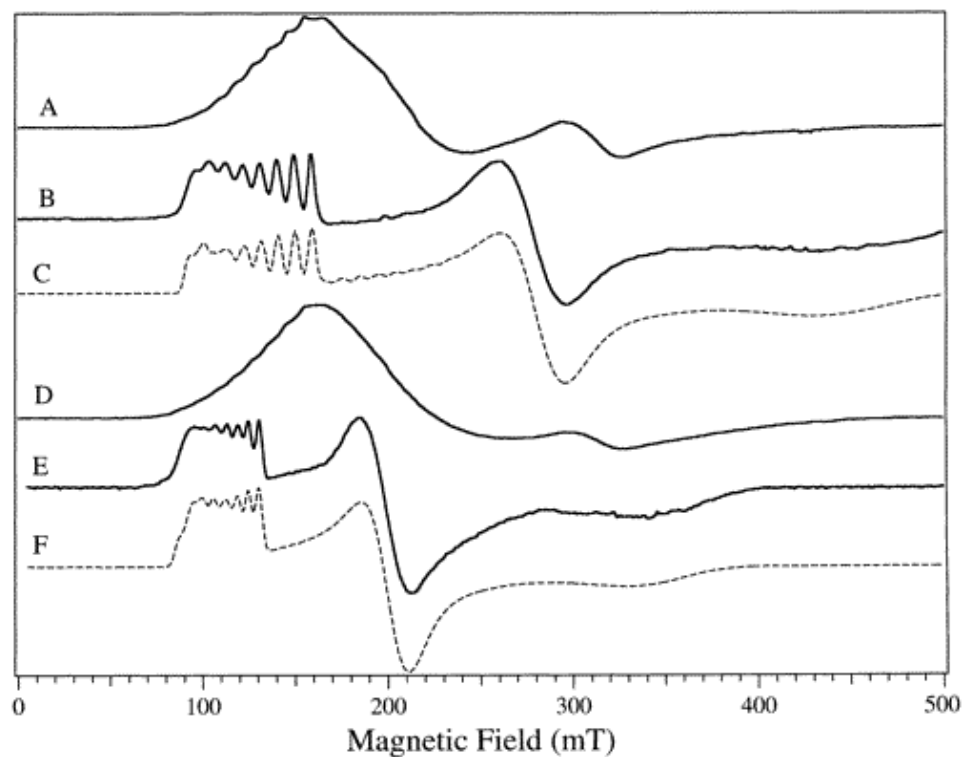

Figure 3 EPR spectra of $(A)$ [CoZn(AAP)] in 50 mM Hepes buffer, pH 7.5, containing 20\% 2-propanol; (B) [CoZn(AAP)] after the addition of 5 equiv of LPA; (C) simulation of (B) with $g_{\text {eff }}=1.90,3.05,6.66, A_{z}\left({ }^{59} \mathrm{Co}\right)=7.0 \mathrm{mT}, M_{\mathrm{s}}=| \pm 1 / 2\rangle, g_{\text {real }}=2.53, E / D$ $=0.26 ;(D)[Z n C o(A A P)]$ in $50 \mathrm{mM}$ Hepes buffer, pH 7.5, containing 20\% 2-propanol; (E) $[\mathrm{ZnCo}(\mathrm{AAP})]$ in the presence of 5 equiv of LPA; and (F) simulation of (E) with $g_{\text {eff }}=$ $1.95,3.40,1.95, A_{z}\left({ }^{59} \mathrm{Co}\right)=4.95 \mathrm{mT}, M_{\mathrm{s}}=| \pm 1 / 2\rangle, g_{\text {real }(x, y)}=2.204$ and $g_{\text {real }(z)}=$ $2.48, E / D=0.2$. All spectra were recorded at $10 \mathrm{~K}, 0.2 \mathrm{~mW}, 1.26 \mathrm{mT}$ modulation amplitude and $10 \mathrm{mT} \mathrm{s}^{-1}$ sweep rate. All EPR simulations were performed with the programs EPRSim XOP and Rhombogram as previously described. ${ }^{10}$

Combination of the optical and EPR data presented herein with the previously reported $\mathrm{X}$-ray crystal structure of $A \mathrm{AP}^{9}$ provides structural information on the transition state of peptide hydrolysis as well as insight into the catalytic mechanism of AAP. Since LPA interacts with both metal centers and a single oxygen atom bridge is likely absent, LPA appears to bind to the dicobalt(II) cluster of AAP as an n$1,2-\mu$-phosphonate with one ligand to the second $\mathrm{Co}$ (II) ion provided by the $\mathrm{N}$-terminal amine. The proposed structure for LPA binding to AAP is consistent with preliminary $\mathrm{X}$-ray crystallographic data for [ZnZn(AAP)] bound by LPA. ${ }^{21}$ On the basis of previously reported kinetic, thermodynamic, spectroscopic, and X-ray crystallographic data a detailed mechanism of action for AAP has recently been proposed. ${ }^{8,10}$ 
In this mechanism, the bridging water $/\left(\mathrm{OH}^{-}\right)$becomes terminal upon substrate binding and is bound to the first $\mathrm{Zn}$ (II) binding site consistent with the fact that (i) AAP is ca. $80 \%$ active with only a single $\mathrm{Zn}$ (II) ion bound, (ii) the substrate-analogue inhibitor 1butaneboronic acid interacts only with the first metal binding site, and (iii) fluoride binding occurs after substrate binding. ${ }^{8,23}$ At this point in the mechanism, the terminal metal bound hydroxide attacks the activated scissile carbonyl carbon of the peptide substrate forming a gem-diolate transition state complex with no single oxygen atom bridge. In addition, $\mathrm{N}$-terminal amine binding to $\mathrm{Zn}_{2}$ suggests that electron density donation by the second metal ion is important in stabilizing the transition state. Amine binding to the second $\mathrm{Mn}$ (II) ion in arginase has been proposed to drive the loss of the bridging water/ $\left(\mathrm{OH}^{-}\right)$to form a terminal hydroxide that functions as the nucleophile in the hydrolytic reaction. ${ }^{24}$ On the basis of these data we propose that $\mathrm{N}$-terminal amine binding, in conjunction with hydrogenbond formation, likely functions to displace the bridging water/ $\left(\mathrm{OH}^{-}\right)$ thus placing it on $\mathrm{Zn}_{1}$ along with the substrate. Therefore, both metal ions are required for full enzymatic activity, but their individual roles appear to differ markedly.

\section{Acknowledgment}

The authors are grateful to Dr. Norbert Sträter of Harvard University for kindly providing the inhibitor I-leucinephosphonic acid. This work was supported by the National Science Foundation (CHE-9422098; R.C.H.). The Bruker ESP-300E EPR spectrometer was purchased with funds provided by the National Science Foundation (BIR-9413530) and Utah State University.

\section{References}

${ }^{1}$ Sträter, N.; Lipscomb, W. N.; Klabunde, T.; Krebs, B. Angew. Chem., Int. Ed. Engl. 1996, 35, 2024-2055.

${ }^{2}$ Lipscomb, W. N.; Sträter, N. Chem. Rev. 1996, 96, 2375-2433.

${ }^{3}$ Wilcox, D. E. Chem. Rev. 1996, 96, 2435-2458.

${ }^{4}$ Dismukes, G. C. Chem. Rev. 1996, 96, 2909-2926.

${ }^{5}$ Griffith, E. C.; Su, Z.; Turk, B. E.; Chen, S.; Chang, Y.-H.; Wu, Z.; Biemann, K.; Liu, J. O. Chem. Biochem. 1997, 4, 461-471.

${ }^{6}$ Sin, N.; Meng, L.; Wang, M. Q.; Wen, J. J.; Bornmann, W. G.; Crews, C. M. Proc. Natl. Acad. Sci. U.S.A. 1997, 94, 6099-6103. 
NOT THE PUBLISHED VERSION; this is the author's final, peer-reviewed manuscript. The published version may be accessed by following the link in the citation at the bottom of the page.

${ }^{7}$ Pulido-Cejudo, G.; Conway, B.; Proulx, P.; Brown, R.; Izaguirre, C. A. Antivir. Res. 1997, 36, 167-177.

${ }^{8}$ Chen, G.; Edwards, T.; D'souza, V. M.; Holz, R. C. Biochemistry 1997, 36, 4278-4286.

${ }^{9}$ Chevrier, B.; Schalk, C.; D'Orchymont, H.; Rondeau, J.-M.; Moras, D.; Tarnus, C. Structure 1994, 2, 283-291.

${ }^{10}$ Bennett, B.; Holz, R. C. J. Am. Chem. Soc. 1997, 119, 1923-1933.

${ }^{11}$ Bennett, B.; Holz, R. C. Biochemistry 1997, 36, 9837-9846.

${ }^{12}$ Bicknell, R.; Holmquist, B.; Lee, F. S.; Martin, M. T.; Riordan, J. F. Biochemistry 1987, 26, 7291-7297.

${ }^{13}$ Moratal, J.-M.; Castells, J.; Donaire, A.; Salgado, J.; Jimenez, H. R. ; Domingo, R. J. Inorg. Biochem. 1994, 53, 1-11.

${ }^{14}$ Bertini, I.; Gerber, M.; Lanini, G.; Luchinat, C.; Maret, W.; Rawer, S. ; Zeppezauer, M. J. Am. Chem. Soc. 1984, 106, 1826-1830.

${ }^{15}$ Turpeinen, U.; Ahlgren, M.; Hämäläinen, R. Acta Crystallogr. 1982, B32, $1580-1583$.

${ }^{16}$ Turpeinen, U.; Hämäläinen, R.; Reedijk, J. Polyhedron 1987, 6, 1603-1610.

${ }^{17}$ Chaudhuri, P.; Ouerbach, J.; Wieghardt, K.; Nuber, B.; Weiss, J. J. Chem. Dalton Trans. 1990, 271-278.

${ }^{18}$ Glerup, J.; Goodson, P. A.; Hodgson, D. J.; Michelsen, K. Inorg. Chem. 1995, 34, 6255-6264.

${ }^{19}$ Kalinnikov, V. T.; Rakitin, Y. V.; Hatfield, W. E. Inorg. Chim. Acta 1978, 31, $1-4$.

${ }^{20}$ Little, I. R.; Straughan, B. P.; Thornton, P. J. Chem. Soc., Dalton. Trans. 1986, 2211-2214.

${ }^{21}$ DePaola, C.; Bennett, B.; Holz, R. C.; Ringe, D.; Petsko, G. Unpublished results.

${ }^{22}$ Chevrier, B.; D'Orchymont, H.; Schalk, C.; Tarnus, C.; Moras, D. Eur. J. Biochem. 1996, 237, 393-398.

${ }^{23}$ Baker, J. O.; Prescott, J. M. Biochemistry 1983, 22, 5322-5331.

${ }^{24}$ Khangulov, S. V.; Sossong, T. M.; Ash, D. E.; Dismukes, G. C. Biochemistry 1998, 37, 8539-8550. 